means were significantly lower in Group B at all ages from 3 to 8, in particular in children from 3 to 6 (Group B: 1.91, sd 1.88, Group A: 3.94, sd 1.54, $\mathrm{p}=0.00001$ ), but not from age 9 to 13 .

Conclusion Our study shows that the Cala-Dol technique is an effective, simple and inexpensive way to reduce pain caused by intramuscular immunization in the deltoid muscle in in children aged 3 to 9 .

\section{SCREENING DEVELOPMENTAL DOMAINS IN PRETERM CHILDREN: DIAGNOSTIC VALIDITY OF "PARENTS' EVALUATION OF DEVELOPMENTAL STATUS: DEVELOPMENTAL MILESTONES" (PEDS:DM) ASSESSMENT LEVEL}

doi:10.1136/archdischild-2012-302724.0414

\begin{abstract}
1.2MA Pritchard, ${ }^{2} \mathrm{~T}$ de Dassel, ${ }^{3} \mathrm{~L}$ Johnston, ${ }^{\mathrm{A}} \mathrm{E}$ Beller, ${ }^{5} \mathrm{~F}$ Bogossian, ${ }^{2} \mathrm{~S}$ Callan, ${ }^{1} \mathrm{C}$ Crothers, ${ }^{6} \mathrm{~S}$ Russo, 'D Hovey, ${ }^{2} \mathrm{D}$ Cartwright. 'Centre for Clinical Research, The University of Queensland; 'ºmen's Newborn Services, Royal Brisbane Women's Hospital, Brisbane, QLD, Australia; ${ }^{3} N u r s i n g$ and Midwifery, Queen's University, Belfast, Ireland; ${ }^{4}$ Centre for Research in Evidence-Based Practice, Bond University, Robina; ${ }^{5}$ Nursing and Midwifery, The University of Queensland; ${ }^{6}$ Better Life Psychology, Brisbane, OLD, Australia
\end{abstract}

Background/aims Very preterm children (VPT-born $<29$ weeks gestation) are at high risk for delay across a range of developmental domains. The diagnostic utility of the Parents' Evaluation of Developmental Status: Developmental Milestones (PEDS:DM) Assessment Level in screening for children with i)domain specific and ii) global cognitive delay was assessed in VPT children.

Method Cross-sectional cohort of infants at 2 and 4-years corrected age for prematurity during 2010. Parents completed the PEDS:DM-Assessment Level in correctly identifying language, motor, self-help and social-emotional domain development which was compared with a blinded 2-year Bayley Scales of Infant Development III, 4-year Wechsler Preschool and Primary Scale of Intelligence-Third Edition and for both ages a Neurosensory Motor Development Assessment and Adaptive Behaviour Assessment System-Second Edition. Diagnostic validity-screening test characteristics were determined for each domain and global cognition.

Results Complete data was available on 149/192 (2-years, N=73 and 4-years, $\mathrm{N}=76$ ) children. The prevalence for developmental delay using each tools standardized mean $->2$ (SD) was lower in all domains and for global-cognition compared to the established cutoff PEDS:DM domain scores ( $\geq 25 \%-16^{\text {th }}$ percentile). Sensitivity and specificity were consistently high (predominately $>70 \%$ ) as was the negative predictive value $(>77 \%)$. The positive predictive value was lower reflecting the high over-referral rate. Mothers stated they found the assessment useful in articulating their infants' developmental strengths and weaknesses by domains.

Conclusion This parent friendly tool has good diagnostic utility for identifying domain specific and global cognitive delay and can be used to enhance surveillance, and would be useful in resource restricted environments.

\section{PARENTS' EVALUATION OF DEVELOPMENTAL STATUS (PEDS:DM) AND THE PARENTING RELATIONSHIP IN CHILDREN BORN VERY PRETERM}

doi:10.1136/archdischild-2012-302724.0415

${ }^{1} \mathrm{MA}$ Pritchard, ${ }^{\mathrm{C}} \mathrm{C}$ Crothers, ${ }^{2 \mathrm{~T}}$ de Dassel, ${ }^{3} \mathrm{~L}$ Johnston, ${ }^{4} \mathrm{E}$ Beller, ${ }^{5} \mathrm{~F}$ Bogossian, ${ }^{2} \mathrm{~S}$ Callan, 'PB Colditz, ${ }^{6} \mathrm{~S}$ Russo, ${ }^{2} \mathrm{D}$ Cartwright. 'Centre for Clinical Research, The University of Queensland; 'Women's Newborn Services, Royal Brisbane Women's Hospital, Brisbane, OLD, Australia; ${ }^{3} N u r s i n g$ and Midwifery, Queen's University, Belfast, Ireland; ${ }^{4}$ Centre for Research in Evidence-Based Practice, Bond University, Robina; ${ }^{5}$ Nursing and Midwifery, The University of Queensland; ${ }^{6}$ Better Life Psychology, Brisbane, OLD, Australia

Background/aim Differences in parenting children born very-preterm (VPT $<29$ weeks gestation) with and without developmental delay has not been fully described. This study examines the association between parent-reported child developmental status, psychosocial-risk and the parenting-relationship in VPT children.

Methods Cross-sectional hospital-cohort of infants at 2 and 4 -years corrected age for prematurity during 2010. Uni-multivariate analysis examined the association between developmental delay (DD) (scores $-\geq 2 \mathrm{SD}$ in $\geq 2$ domains-gross/fine motor, receptive/expressive language, social-emotional and self-help using Parent Evaluations of Developmental Status-Developmental Milestones-(PEDS:DM)-Assessment-Level) and parenting-relationship (score $-\geq 1 S D$ in $\geq 1$ domain-attachment, involvement, discipline-practices, parenting confidence and relational frustration assessed by the Parenting Relationship Questionnaire). Outcomes are for the total cohort and by age-group, adjusted for psychosocial-risk ( $\geq 4 / 11$ risks on Brigance Observations of Psychosocial-Risk Scale).

Results Cohort data is available on 165/192, 86\% (2-years, N=80; 4 -years, $N=85$ ) children which showed parenting a child with DD was associated with higher parenting-relationship problems for the total cohort (OR 3.2, 95\%CI 1.5, 7.0, p<0.01) who experienced greater difficulties in attachment (OR 3.2, 95\%CI 1.1, 10.3, p =0.04) and parenting confidence (OR 4.7, 95\%CI, 2.1, 11.5, p<0.01) compared to the non-delayed group. Differences by age group were (2-year-group, attachment; OR 7.3, 95\%CI 1.4, 37.0, p =0.02) and (4-year-group, parenting confidence; OR 16.0, 95\%CI 4.4, 57.3, $\mathrm{p}<0.01)$ found.

Conclusion Mothers of VPT children with DD may require additional parenting support; strengthening early attachment may impact latter parenting confidence in these families. Examining these findings in relation to child behavior and possible pathways for intervention is planned.

\section{IMPACT OF EXERCISE ON LOWER ACTIVITY LEVELS IN CHILDREN WITH ACUTE LYMPHOBLASTIC LEUKEMIA: A RANDOMIZED CONTROLLED TRIAL FROM TURKEY}

doi:10.1136/archdischild-2012-302724.0416

'M Kurtuncu Tanir, ${ }^{2}$ S Kuguoglu. 'Pediatric Nursing, Zonguldak Karaelmas University, Zonguldak, Turkey; ${ }^{2}$ Pediatric Nursing, Emeritus Professor from Marmara University, Brooklyn, NY, USA

Background and Aims Children with Acute lymphoblastic leukemia (ALL) exhibit body function disorders and activity limitations at an early stage. A study assessing the impact of activities and health-related quality of life of initiatives physical exercise in children with ALL were not found in Turkey. This study was carried out to determine the effects of an exercise program on both physical parameters and on quality of life in children with ALL.

Methods A total of 41 children with ALL at two university hospitals were accepted into the study. Due to the demise of one of the children in the trial group, the study was completed with 19 trial and 21 control patients, a total of 40 children and their parents. The two groups were formed by randomized selection. The study was implemented in the children's homes and in the clinical environment and in the period 2007-2008.

Results When the trial subjects were assessed in terms of their mean scores in the 9-Minute Walk Test, the Timed Up and Down Stairs Test, the Timed Up and Go Test, the measurements of their leg muscle strength, their hemoglobin and hematocrit tests, a decidedly significant increase was seen compared to the control group $(\mathrm{p}<0.05)$.

Conclusions Regular and systematic exercise regimens implemented by children with ALL have resulted in improved testing results, enhanced physical performance, and better laboratory results compared to a control group and to children's scores prior to the initiation of such a program. 